\title{
Oscillator damped by a constant-magnitude friction force
}

\author{
Avi Marchewka ${ }^{\text {a) }}$ \\ Department of Physics, North Carolina State University, Raleigh, North Carolina 27695-8202 \\ David S. Abbott ${ }^{\text {b) }}$ \\ Dartmouth College, New Hanover, New Hampshire 03755 \\ Robert J. Beichner ${ }^{\mathrm{c}}$ \\ Department of Physics, North Carolina State University, Raleigh, North Carolina 27695-8202
}

(Received 27 February 2003; accepted 15 September 2003)

\begin{abstract}
Although a simple spring/mass system damped by a friction force of constant magnitude shares many of the characteristics of the simple and damped harmonic oscillators, its solution is not presented in most texts. Closed form solutions for the turning and stopping points can be found using an energy-based approach. A dynamical approach leads to a closed form solution for the position of the mass as a function of time. The main result is that the amplitude of the oscillator damped by a constant magnitude friction force decreases by a constant amount each swing and the motion dies out after a finite time. We present these two solutions and suggest ways that the system can be used in the classroom. (C) 2004 American Association of Physics Teachers.
\end{abstract}

[DOI: $10.1119 / 1.1624113$ ]

\section{INTRODUCTION}

Introductory courses in physics strive to teach students to think physically and become better problem solvers. Interactive studio physics classes have been shown to improve students' conceptual understanding and problem-solving ability. ${ }^{1-3}$ In such classes, students typically work together to solve carefully constructed problems. These problems range from conceptual exercises like those presented in Ref. 4 to context-rich quantitative problems. ${ }^{5}$ Some studio classes also integrate computers into the curriculum. In the SCALE-UP ${ }^{6}$ classroom at North Carolina State University, students use computer tools such as Interactive Physics ${ }^{7}$ and VPython ${ }^{8}$ to model the behavior of physical systems and to bridge the gap between graphical, symbolic, and visual representations. This article focuses on a problem studied in the SCALE-UP classroom that can be used to improve students' problemsolving and computer modeling skills.

The physical system is easy to describe: a block resting on a rough horizontal surface is attached to a stretched spring and released. Figure 1 shows how the problem was presented to the students at the beginning of the simple harmonic motion unit. The question was intended as a simple application of the work-energy theorem. However, the students (and instructors) quickly discovered that the addition of a constant frictional force made the system considerably richer than either had imagined. Although the spring/mass system often is presented in the context of simple harmonic oscillators, the spring/mass system damped by a force of constant magnitude is rarely studied. Because the problem is not presented in any readily available text, ${ }^{9}$ students cannot use a formula matching approach or mimic a textbook solution.

The system can be solved analytically, but the solution is not easy. Students must start from first principles, reason qualitatively to plan a solution strategy, and then check the qualitative behavior of the result. The system has few components, so it can be easily simulated. Using the computer, students can check their hunches about the system's behavior or perform controlled simulations to explore the effect of changing system parameters. Because the system is closely related to the simple and damped harmonic oscillators, the system includes widely applicable physics and shares many of the characteristics of more commonly studied oscillators. However, as we will show, the motion of the system is unique in several key ways and may provide insight into the dynamics of real damped systems. In the following we present two general solutions for the oscillator damped by a constant magnitude force and suggest ways that the problem might be used in the physics classroom.

\section{THE MODEL SYSTEM}

A block of mass $m$ attached to a spring of strength $k$ moves on a rough horizontal surface with friction coefficients, $\mu_{s} \geqslant \mu_{k}$. The block is released from rest. For convenience, the origin of the coordinates is chosen so that the spring has minimum potential energy at the origin. A cursory look at the system strongly suggests that the mass will eventually stop moving, but it is not immediately clear when (or where) this will happen. Indeed, students in our classes asked many of the salient questions: When will the motion stop? How far will the mass travel before it stops? How many times will the mass go back and forth? Where (at what position) will the mass stop? These questions cannot be answered in one or two steps. The answers depend on how far the block is from the origin when it is released. If the block starts very close to the origin, the block will not move at all. Start a little further from the origin, and the block will break free and slide, but may not be able to move again when it comes to rest at the far end of its motion.

\section{ENERGY-LIKE METHODS: AN ALGEBRAIC ANALYSIS}

Before diving into the details, the problem solver must map a strategy for solving the problem based on fundamental physics concepts. ${ }^{10}$ The qualitative analysis offered in the previous paragraph suggests one strategy for solving this problem. Figure 2 shows a flowchart for an iterative solution employing the principle of energy conservation. Heuristics 


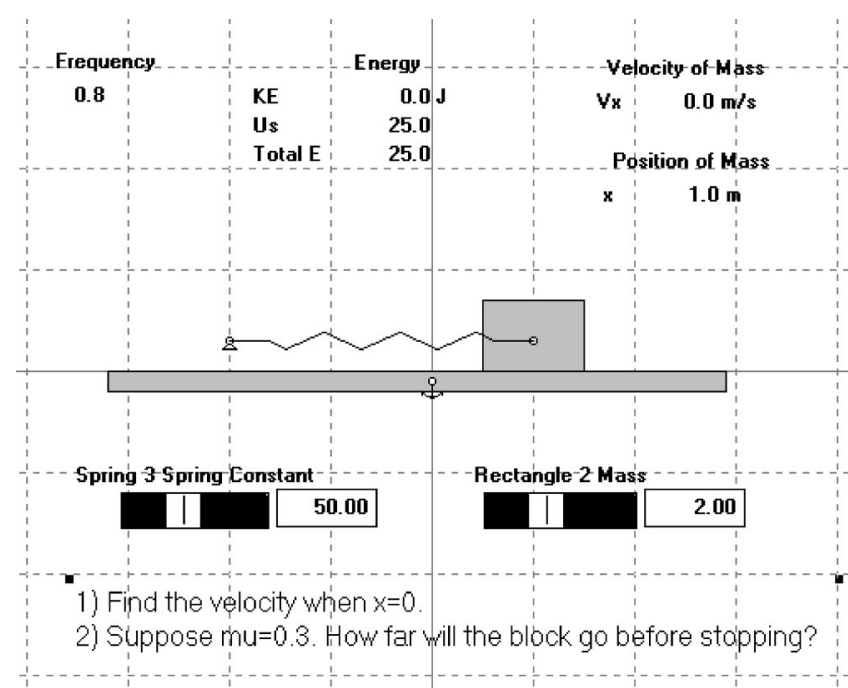

Fig. 1. The problem presented to students in the SCALE-UP class. The instructor displayed this static image, which was created in a simulation package, Interactive Physics. Once students solved the problem, they were given access to the simulation file. In the simulation program, students can use the sliders to alter system parameters. Click-dragging the box alters its initial position.

such as the flowchart in Fig. 2 often are employed by expert problem solvers, but are rarely useful for solving the types of problems presented in most textbooks.

To construct the solution, we follow the flowchart. Assume that the block is momentarily at rest at position $x_{n}$. If the spring force does not exceed the force of static friction (that is, if $k\left|x_{n}\right| \leqslant \mu_{s} m g$ ), the motion ceases. Otherwise, the block will break free and slide. The block will travel toward the origin and eventually come to rest, at least momentarily, at some new position, $x_{n+1}$. The position of these two turning/ stopping points, $x_{n+1}$ and $x_{n}$, can be easily related using

$$
\int F_{\text {tot }} d r_{\text {c.m. }}=\Delta\left(\frac{1}{2} m v_{\mathrm{c} . \mathrm{m} .}^{2}\right),
$$

where $F_{\text {tot }}$ is the sum of the external forces acting on the block.

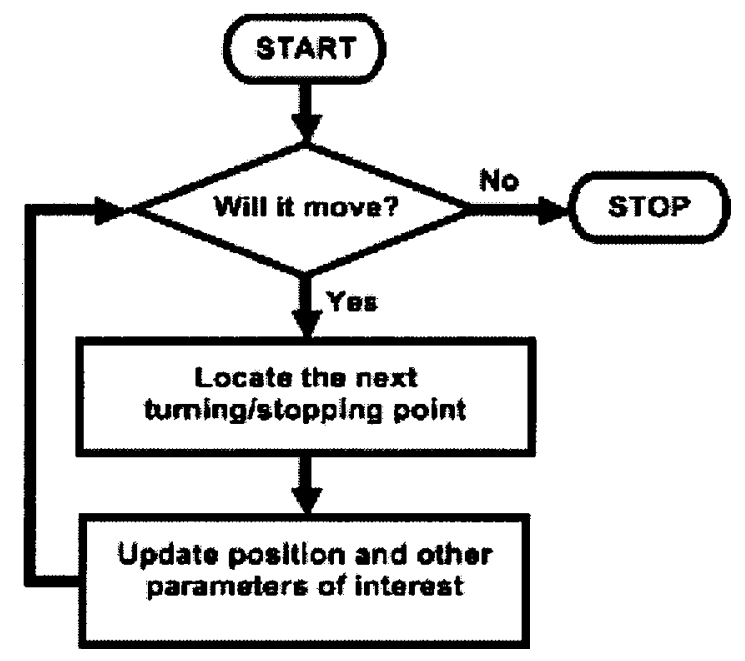

Fig. 2. The flowchart shows one strategy for creating a map of the motion that can be used to find the total distance traveled by the object.
Before continuing, a few words about Eq. (1) are in order. Although Eq. (1) is sometimes called the work-energy theorem, this name is somewhat misleading. If parts of an extended body move relative to the center of mass, the integral on the left-hand side of Eq. (1) is not equal to the total work done on the body. Likewise, the kinetic energy-like term on the right-hand side is not the total kinetic energy of the body, because it excludes contributions from parts of the body that are moving relative to the center of mass. As a result, some physicists refer to the integral $\int F_{\text {tot }} d r_{\text {c.m. }}$ as pseudowork and Eq. (1) as the center of mass equation. Though it rarely affects getting the right answer for traditional mechanics problems like the one presented here, failure to carefully distinguish between different "works" and "energies" can lead to sloppy reasoning about energy and wrong answers in other contexts (for example, thermal physics). For a fuller discussion of work, pseudowork, and related issues, see Refs. 11 or 12.

If we use Eq. (1) to relate the position of one stopping/ starting point and the next, we obtain

$$
\frac{1}{2} k x_{n+1}^{2}-\frac{1}{2} k x_{n}^{2}-\mu_{k} m g\left|x_{n+1}-x_{n}\right|=0 .
$$

As the absolute value sign suggests, there are two cases to be considered when solving for $x_{n+1}$. If the starting/stopping point is beyond some critical distance from the origin, the block will have to cross the origin and the positions of the turning/stopping points, $x_{n+1}$ and $x_{n}$, have the opposite algebraic signs. If the block is less than this critical distance from the origin, $x_{n+1}$ and $x_{n}$ are on the same side of the origin and have the same sign.

Same Side Motion (Case I). In this case, the starting position $x_{n}$ is far enough from the origin that the block will move, but close enough to the origin that the mass will not cross the origin. In this case, $x_{n+1}$ and $x_{n}$ have the same sign and $\left|x_{n+1}-x_{n}\right|$ can be replaced by $\left|x_{n+1}\right|-\left|x_{n}\right|$. Equation (2) becomes:

$$
\frac{1}{2} k x_{n+1}^{2}-\frac{1}{2} k x_{n}^{2}=\mu_{k} m g\left(\left|x_{n+1}\right|-\left|x_{n}\right|\right) .
$$

Equation (3) can be solved for $\left|x_{n+1}\right|$ by factoring the lefthand side and then simplifying:

$$
\frac{1}{2} k\left(\left|x_{n+1}\right|+\left|x_{n}\right|\right)\left(\left|x_{n+1}\right|-\left|x_{n}\right|\right)=\mu_{k} m g\left(\left|x_{n+1}\right|-\left|x_{n}\right|\right),
$$

$$
\left|x_{n+1}\right|+\left|x_{n}\right|=\frac{2 \mu_{k} m g}{k}
$$

or

$$
\left|x_{n+1}\right|=C-\left|x_{n}\right|,
$$

where $C \equiv 2 \mu_{k} m g / k$. Same side motion can occur only if the maximum pseudowork the spring can do does not exceed the pseudowork needed to move the block past the origin against friction $\left(\frac{1}{2} k x_{n}^{2} \leqslant \mu_{k} m g\left|x_{n}\right|\right)$. It is not surprising that this condition reduces to $\left|x_{n}\right| \leqslant C$, because Eq. (6) has no solution if $\left|x_{n}\right|>C$. Thus, $C$ represents a characteristic distance for this system.

Not only must the turning/stopping point $x_{n}$ be within a distance $C$ of the origin for same side motion to occur, it must be far enough from the origin for movement to occur. For this to happen, the spring force must exceed the static friction force at the turning/stopping point $\left(k\left|x_{n}\right|>\mu_{s} m g\right)$. 
We can rewrite this condition to obtain a characteristic distance related to the static coefficient of friction:

$$
\left|x_{n}\right|>D
$$

where $D \equiv \mu_{s} m g / k$. In order for same side motion to occur, $C \geqslant\left|x_{n}\right|>D$. Note that same side motion is impossible if $\mu_{s}>2 \mu_{k}$, because $D$ must be larger than $C$ for the condition $C \geqslant\left|x_{n}\right|>D$ to hold. It is interesting that, up to dimensionless factors, the values of these two characteristic distances can be obtained from dimensional analysis.

Intuition suggests that same side motion occurs at most once. It seems reasonable that if the mass can't cross the origin, the spring will not be stretched enough at $x_{n+1}$ to overcome static friction and the mass will remain at rest when it reaches $x_{n+1}$. Let's check this supposition. If the mass fails to cross the origin, $\left|x_{n+1}\right|=C-\left|x_{n}\right|$ and $\left|x_{n+1}\right|$ $<\left|x_{n}\right|$, so that $\left|x_{n+1}\right|<\frac{1}{2} C$ or, equivalently, $k\left|x_{n+1}\right|$ $<\mu_{k} m g$. Because the spring force is weaker than the force of kinetic friction, the spring force will be weaker than the force of static friction, provided that $\mu_{s} \geqslant \mu_{k}$. Thus the block will not move when it reaches $x_{n+1}$.

Origin Crossing Motion (Case II). In this case, the mass starts far enough from the origin that it will cross the origin when it travels from one turning/stopping point to the next. Now $x_{n+1}$ and $x_{n}$ have opposite signs and $\left|x_{n+1}-x_{n}\right|$ can be replaced by $\left|x_{n+1}\right|+\left|x_{n}\right|$. In this case, Eq. (2) reduces to

$$
\left|x_{n+1}\right|=\left|x_{n}\right|-C \text {. }
$$

Equation (2) now yields a recursion relation. Each time the mass passes through the origin, the turning/stopping point moves a distance $C$ closer to the origin. Notice that this linear recursion relation is in sharp contrast with the exponential decay of an oscillator damped by a force proportional to velocity. The iterative application of Eq. (8) shows that

$$
\left|x_{n+1}\right|=\left|x_{0}\right|-(n+1) C,
$$

where $x_{0}$ is the initial position of the mass and $x_{n+1}$ is the position after $n+1$ trips through the origin. Equations (8) and (9) remain valid as long as both the condition for crossing the origin $\left(\left|x_{n}\right|>C\right)$ and the condition for movement $\left(\left|x_{n}\right|>D\right)$ hold. With each trip through the origin, the turning/stopping point moves a distance $C$ closer to the origin. The pattern continues until one of these two conditions fails.

For a few combinations of surfaces, such as glass on glass, ${ }^{13} \mu_{s}>2 \mu_{k}$ (or, equivalently, $D>C$ ). For these combinations, the condition for movement fails before the condition for crossing the origin can fail. The substitution of Eq. (9) into the condition for movement determines the upper bound on $n$ :

$$
\left|x_{0}\right|-n C>D \quad\left(\mu_{s}>2 \mu_{k}\right) .
$$

If we solve for $n$ and use the definition of $D$, we find

$$
n<\frac{\left|x_{0}\right|}{C}-\frac{\mu_{s}}{2 \mu_{k}} \quad\left(\mu_{s}>2 \mu_{k}\right) .
$$

Equation (11) means that the mass goes through the origin $N$ times, where

$$
N=1+\operatorname{int} *\left(\frac{\left|x_{0}\right|}{C}-\frac{\mu_{s}}{2 \mu_{k}}\right) \quad\left(\mu_{s}>2 \mu_{k}\right),
$$

and int* denotes the largest integer less than the argument. ${ }^{14}$ (Remember that the mass crosses the origin once when it moves from $x_{n}$ to $x_{n+1}$.) For these combinations of surfaces with $\mu_{s}>2 \mu_{k}$, motion ceases completely when the mass arrives at $x_{N+1}$, because same side motion is impossible if $\mu_{s}>2 \mu_{k}$.

For most combinations of surfaces, however, $\mu_{s} \leqslant 2 \mu_{k}$, and the requirement that $\left|x_{n}\right|>C$ fails before the condition for movement can fail. In this regime, we can substitute Eq. (9) into $\left|x_{n}\right|>C$ to determine the number of trips through the origin. The result is that

$$
N=\operatorname{int} *\left(\frac{\left|x_{0}\right|}{C}\right) \quad\left(\mu_{s} \leqslant 2 \mu_{k}\right) .
$$

In this domain where $\mu_{s} \leqslant 2 \mu_{k}$, the end of origin crossing motion does not necessarily signal the end of motion. After $N$ origin crossings, the mass arrives at $x_{N}$. If $k\left|x_{N}\right|>\mu_{s} m g$, the block will execute same side motion before motion ceases. This occurs if $\left|x_{N}\right|>D$ or

$$
\frac{\left|x_{0}\right|}{C}-\mathrm{int} *\left(\frac{\left|x_{0}\right|}{C}\right)>\frac{\mu_{s}}{2 \mu_{k}} \text {. }
$$

Equation (14) is found by substituting the results from Eqs. (6), (7), and (13) into $\left|x_{N+1}\right|>D$. Notice that condition (14) is more restrictive than $\mu_{s} \leqslant 2 \mu_{k}$, because the left-hand side must be less than unity. Equation (14) reflects the reality that $\mu_{s} \leqslant 2 \mu_{k}$ constitutes a necessary, but not sufficient, condition for same side motion.

The total distance traveled by the mass can now be calculated. The mass will execute $N$ trips through the origin. If the conditions are just right, the object will execute same side motion and stop. Otherwise, the motion ceases immediately after the $N$ trips through the origin. The distance traveled during the origin crossings can be found by brute force summation. Because the first trip through the origin starts from position $x_{0}$,

$$
S_{I I}=\sum_{n=1}^{N} S_{n-1}=\sum_{n=1}^{N}\left(\left|x_{n-1}\right|+\left|x_{n}\right|\right) .
$$

If we use Eq. (9) twice, once with $n-1$ and once with $n$, Eq. (15) becomes

$$
\begin{aligned}
S_{I I}=\sum_{n=1}^{N} S_{n-1} & =\sum_{n=1}^{N}\left(\left|x_{0}\right|-(n-1) C+\left|x_{0}\right|-n C\right) \\
& =\sum_{n=1}^{N}\left(2\left|x_{0}\right|+C-2 n C\right),
\end{aligned}
$$

or, simplifying the sum,

$$
S_{I I}=2 N\left|x_{0}\right|-N^{2} C \text {. }
$$

Equation (17) can also be found more elegantly by applying the relation between pseudowork and center of mass kinetic energy:

$$
-\mu_{k} m g S_{I I}-\frac{1}{2} k\left(\left|x_{N}\right|^{2}-\left|x_{0}\right|^{2}\right)=0 .
$$

Unless the object executes same side motion, the subtotal, $S_{I I}$, represents the total distance traveled. If condition (14) holds, same side motion occurs and the mass travels an additional distance, $S_{I}$, before stopping:

$$
S_{I}=\left|x_{N}\right|-\left|X_{N+1}\right| \text {. }
$$


Table I. Summary of results for the algebraic solution for different ranges of $\mu_{s}$. The number of times the mass crosses the origin, $N$, and the total distance traveled, $S$, depend on the characteristic distance that measures the relative strength of the spring force and the kinetic friction force: $C$ $\equiv 2 \mu_{k} \mathrm{mg} / \mathrm{k}$. The function mod represents the remainder of the argument once the integer part has been subtracted: $\bmod (x) \equiv x-\operatorname{int}(x)$.

\begin{tabular}{|c|c|c|}
\hline Range of validity & $\begin{array}{l}\text { Number of origin } \\
\text { crossings, } N\end{array}$ & $\begin{array}{c}\text { Total distance traveled, } \\
S\end{array}$ \\
\hline$\frac{\mu_{s}}{2 \mu_{k}}>1$ & $1+\operatorname{int} *\left(\frac{\left|x_{0}\right|}{C}-\frac{\mu_{s}}{2 \mu_{k}}\right)$ & $2 N\left|x_{0}\right|-N^{2} C$ \\
\hline $1>\frac{\mu_{s}}{2 \mu_{k}}>\bmod \left(\frac{\left|x_{0}\right|}{C}\right.$ & $\operatorname{int}^{*}\left(\frac{\left|x_{0}\right|}{C}\right)$ & $2 N\left|x_{0}\right|-N^{2} C$ \\
\hline $\bmod \left(\frac{\left|x_{0}\right|}{C}\right)>\frac{\mu_{s}}{2 \mu_{k}}$ & $\operatorname{int}^{*}\left(\frac{\left|x_{0}\right|}{C}\right)$ & $2(N+1)\left|x_{0}\right|-(N+1)^{2} C$ \\
\hline
\end{tabular}

If we use Eqs. (6) and (9), Eq. (19) becomes

$$
S_{I}=\left|x_{N}\right|-\left(C-\left|x_{N}\right|\right)=2\left|x_{N}\right|-C=2\left|x_{0}\right|-(2 N+1) C .
$$

If same side motion occurs, the total distance traveled, $S$ $=S_{I}+S_{I I}$, is given by

$$
S=2(N+1)\left|x_{0}\right|-(N+1)^{2} C .
$$

Regardless of the value of initial conditions, the motion terminates after a finite number of passes through the origin. The results of the solution determined so far are summarized in Table I.

There is reason to believe that the three regimes in Table I may be related. Physically, a single condition $\left(k\left|x_{n}\right| \leqslant \mu_{s} m g\right)$ determines when motion terminates. The only physical difference between same side motion and origin crossing motion is where the motion takes place. Mathematically, there are striking similarities across the rows of Table I. It seems plausible that single expressions for $S$ and $N$ may apply for all values of $\mu_{s} \geqslant \mu_{k}$. This possibility will be explored in Sec. IV.

\section{DYNAMICAL ANALYSIS USING DIFFERENTIAL EQUATIONS}

We now find the equation of motion for the mass starting from Newton's second law and gain insight into the dynamics of the system that the pseudowork method presented in Sec. III does not provide. However, the differential equations put the analysis beyond the reach of most students enrolled in introductory physics courses.

Applying Newton's second law to the mass yields one of two linear differential equations for the position of the block, depending on the velocity of the block:

$$
m \ddot{x}=-k x+\mu_{k} m g \quad(\ddot{x}<0)
$$

and

$$
m \ddot{x}=-k x-\mu_{k} m g \quad(\ddot{x}>0) .
$$

The general solution to Eq. (22) is simply the solution to the homogenous equation for the simple harmonic oscillator plus an additive constant:

$$
x_{G}(t)=A \cos (\omega t-\phi)+C / 2 \quad(\ddot{x}<0)
$$

and

$$
x_{G}(t)=A \cos (\omega t-\phi)-C / 2 \quad(\ddot{x}>0),
$$

where

$$
\omega \equiv \sqrt{\frac{k}{m}},
$$

and

$$
C \equiv \frac{2 \mu_{k} m g}{k} .
$$

These general solutions, $x_{G}(t)$, cannot constitute the solution for the equation of motion without modification. There are two major problems. If $A$ represents a single number, the position function is discontinuous at each turning point. Furthermore, if $A$ is a constant for the entire motion, the amplitude of the motion will never decrease. What the general solutions represent is a family of solutions. Each member solution is valid from one turning/stopping point until the next and each has its own value for the constant $A$.

From the form of the general solutions, it is apparent that every half swing exhibits simple harmonic motion. Turning/ stopping points are regularly spaced in time, occurring at a frequency of $2 \omega$. The initial conditions at each turning point determine the amplitude and phase for the following half swing. The solution to the equation of motion is constructed piecemeal from the requirement that the position of the mass, $x(t)$, be a continuous function of time. For simplicity, we will choose the initial conditions so that the block starts with zero velocity at $t=0$. We also will assume that the block is released from a position sufficiently far to the right of the origin that the block breaks free and begins to slide. With this choice of initial conditions, the $n$th turning/stopping point occurs at $t=t_{n}=(\pi / \omega) n$. During the time interval between $t_{n}$ and $t_{n+1}$,

$$
x(t)=A_{n} \cos (\omega t)+(-1)^{n} C / 2,
$$

and

$$
\dot{x}(t)=-\omega A_{n} \sin (\omega t) .
$$

The amplitude, $A_{n}$, must be positive in order for the velocity to have the correct sign for all values of $n$. The position of the $n$th turning point is directly related to the amplitude by substituting $t_{n}=(\pi / \omega) n$ into Eq. (25):

$$
x_{n}=(-1)^{n}\left(A_{n}+C / 2\right) \text {. }
$$

The recursion relation for $A_{n}$ can be obtained by ensuring that $x(t)$ is continuous, that is,

$$
\lim _{t \rightarrow t_{n+1}^{+}} x(t)=\lim _{t \rightarrow t_{n+1}^{-}} x(t) .
$$

Because the limit on the left-hand side is approached from times greater than $t_{n+1}, n$ must be replaced by $n+1$ in Eq. (25). However, Eq. (25) applies to the limit on the right-hand side, so the continuity condition becomes

$$
\begin{aligned}
& A_{n+1} \cos [(n+1) \pi]+(-1)^{n+1} C / 2 \\
& \quad=A_{n} \cos [(n+1) \pi]+(-1)^{n} C / 2, \\
& A_{n+1}(-1)^{n+1}+(-1)^{n+1} C / 2 \\
& \quad=A_{n}(-1)^{n+1}+(-1)^{n} C / 2, \\
& A_{n+1}=A_{n}-C,
\end{aligned}
$$

so that 


$$
A_{n}=A_{0}-n C \text {. }
$$

The physical interpretation of Eq. (31) is that the amplitude of the half swing decreases by $C$ with each half swing. This result matches that of the pseudowork method presented in Sec. III. Moreover, if we substitute the expression for $A_{n}$ from Eq. (27) into Eq. (32), we recover the recursion relation for $x_{n}$ presented in Sec. III.

Equation (32) sets a conservative upper bound on the index $n$, because $A_{n}$ must be a positive number for all $n$. The motion will terminate even sooner if the spring cannot overcome the static force of friction at the turning/stopping point. Motion is forbidden if $k\left|x_{n}\right| \leqslant \mu_{s} m g$. This condition can be rewritten using the definition of $C$ and Eq. (27):

$$
\left|A_{n}+\frac{C}{2}\right| \leqslant \frac{\mu_{s}}{2 \mu_{k}} C \text {. }
$$

Because $A_{n}$ and $C$ are both positive, the absolute value can be removed. If we substitute Eq. (32) into Eq. (33) and rearrange the terms, we find the forbidden values of $n$ :

$$
\begin{aligned}
& A_{0}-n C+\frac{C}{2} \leqslant \frac{\mu_{s}}{2 \mu_{k}} C, \\
& A_{0}+\frac{C}{2}-\frac{\mu_{s}}{2 \mu_{k}} C \leqslant n C, \\
& \frac{A_{0}}{C}-\frac{\mu_{s}-\mu_{k}}{2 \mu_{k}} \leqslant n .
\end{aligned}
$$

Hence, the motion terminates on the index, $n_{\text {stop }}$, given by

$$
n_{\text {stop }}=\operatorname{int} *\left(\frac{A_{0}}{C}-\frac{\mu_{s}-\mu_{k}}{2 \mu_{k}}\right) .
$$

Because the mass stops at the end of the $n$th half swing, the mass executes $\left(n_{\text {stop }}+1\right)$ half swings before stopping. During each half swing, the mass travels a distance $2 A_{n}$, so the total distance traveled is given by

$$
\begin{aligned}
S=2 \sum_{n=0}^{n_{\text {stop }}} A_{n} & =2 \sum_{n=0}^{n_{\text {stop }}}\left(A_{0}-n C\right) \\
& =2\left(n_{\text {stop }}+1\right) A_{0}-n_{\text {stop }}\left(n_{\text {stop }}+1\right) C .
\end{aligned}
$$

At first glance, these results appear to contradict the results of the pseudowork solution, which suggest three different regimes of behavior, depending on the relative values of $\mu_{s}$ and $\mu_{k}$. These regimes, however, are an artifact of the distinction between same side and origin crossing motion. Equation (27) can be used to show that each of the termination conditions given in Table I is equivalent to Eq. (37). Similarly, both expressions for the total distance traveled are equivalent to Eq. (38).

To summarize the results of this section, the motion of each half swing is essentially the motion of a simple harmonic oscillator centered about either $x=-C / 2$ or $x=+C / 2$. At each turning point, the amplitude of the oscillator decreases by $C$ and the center of the oscillatory motion jumps instantaneously from one side of the origin to the other. Before $t=\left(n_{\text {stop }}+1\right) \pi / \omega$, the mass moves according to

$$
x(t)=\left(A_{0}-n C\right) \cos (\omega t)+(-1)^{n} C / 2,
$$

where

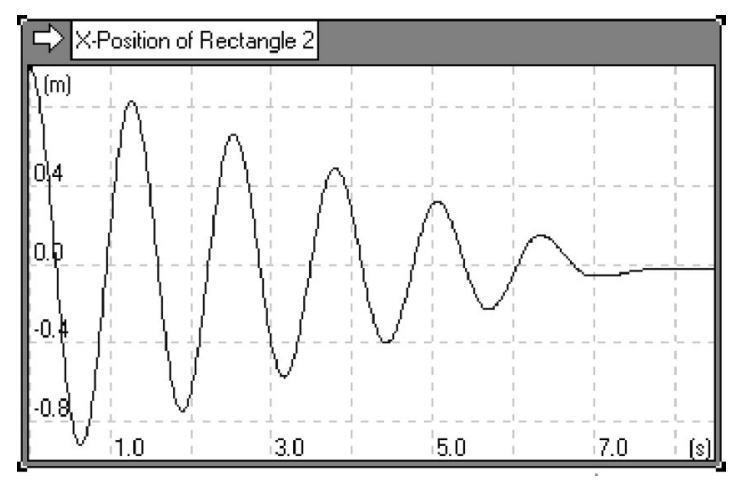

Fig. 3. Graphical output from Interactive Physics for a spring/mass system damped by a constant magnitude force. For this graph, $m=2 \mathrm{~kg}, k$ $=50 \mathrm{~N} / \mathrm{m}, \mu_{s}=0.15, \mu_{k}=0.11$, and $x_{0}=1 \mathrm{~m}$.

$$
n=\operatorname{int}\left(\frac{\omega t}{\pi}\right) .
$$

After $t=\left(n_{\text {stop }}+1\right) \pi / \omega$, the mass remains motionless at

$$
x_{\text {stop }}=(-1)^{n_{\text {stop }}+1}\left[A_{0}-n_{\text {stop }} C\right]+(-1)^{n_{\text {stop }} C / 2 .} \text {. }
$$

Most textbooks treat an oscillator damped by a force proportional to the velocity. The system studied in this article differs from the traditional damped harmonic oscillator in two fundamental ways. The amplitude of the motion decays arithmetically, rather than exponentially. The amplitude, which is constant for each half swing of the motion, decreases by a fixed amount at each turning point. As a result, the motion ceases completely after a finite time, in direct contrast to the traditional damped harmonic oscillator, which never dies out completely.

\section{SIMULATION SOFTWARE}

Even when a detailed mathematical analysis is inappropriate, students can use a variety of software packages to simulate the behavior of real systems and develop an intuitive appreciation of their behavior. For example, with Interactive Physics students can use icons and menus to set up a system to study. Once the parameters have been chosen, the software animates the evolution of the system. Students can experiment with parameters like the spring constant or initial position, to examine how they affect the system's behavior. The

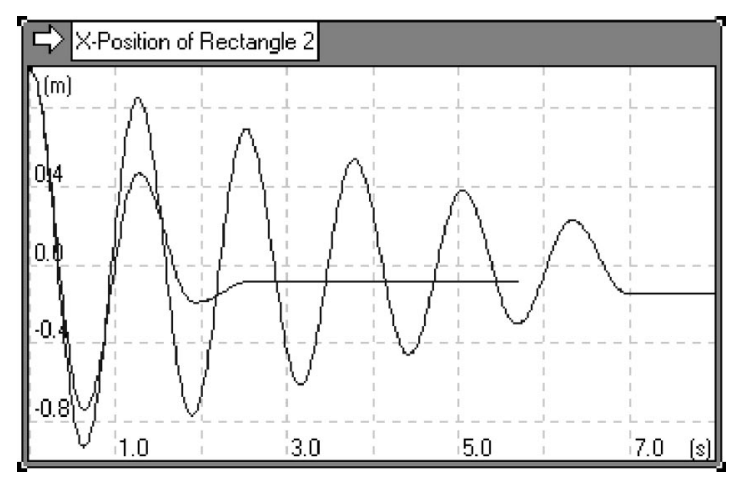

Fig. 4. Comparison of the evolution of two spring/mass systems damped by a constant magnitude force for two different values of $\mu_{k}: 0.1$ and 0.35 . The systems share other system parameters: $m=2 \mathrm{~kg}, k=50 \mathrm{~N} / \mathrm{m}, \mu_{s}=0.4$, and $x_{0}=1 \mathrm{~m}$. 
Fig. 5. A VPython program that produces an animation of the system while it generates a position vs time graph for the block. Screen shots of the program's output are shown in Figs. 6 and 7. program also can produce graphs which are displayed in real time alongside the animated system. Figure 3 shows a graph generated by Interactive Physics to check qualitative and quantitative features of the analytical solutions presented in this paper. The display shows the qualitative features, such as the sinusoidal time dependence and linear envelope. Several runs can be displayed on the same graph, so that the effect of varying system parameters can be studied. Figure 4 explores the effect of the kinetic coefficient on the time evolution of the system. Controlled comparisons, such as the one suggested by Fig. 4, can be used before a student has done any mathematical analysis to test ideas about the system as well as develop students' experimentation skills. Graphs and numerical output also can be used to check quantitative predictions once a solution has been produced. Indeed, the authors used Interactive Physics to check many key results of the analysis presented in this paper.

The drawback of using microworld packages like Interactive Physics is that the computer calculates the solution for the problem. All the student has to do is set up the system and the computer takes care of all the physics. An alternative is an environment where the student must program the physics into the simulation. The danger of using programming environments is that the physics can be obscured by the cognitive requirements of programming. To combat this problem, physics educators and programmers have developed VPython. VPython retains the features of the Python programming language, but also includes modules that automate output tasks helpful in the physics classroom. As a result, student effort can be focused on physics, instead of on programming the visualization tools.

Two teachers at North Carolina State University (Ruth Chabay and Bruce Sherwood) use VPython extensively in the SCALE-UP classroom. Their text, Matter and Interactions, ${ }^{15}$ is an excellent source of programming-based problems. In the mechanics part of the matter and interactions curriculum, students routinely write programs to analyze the motion of physical systems. This approach highlights the primacy of Newton's second law in mechanics,

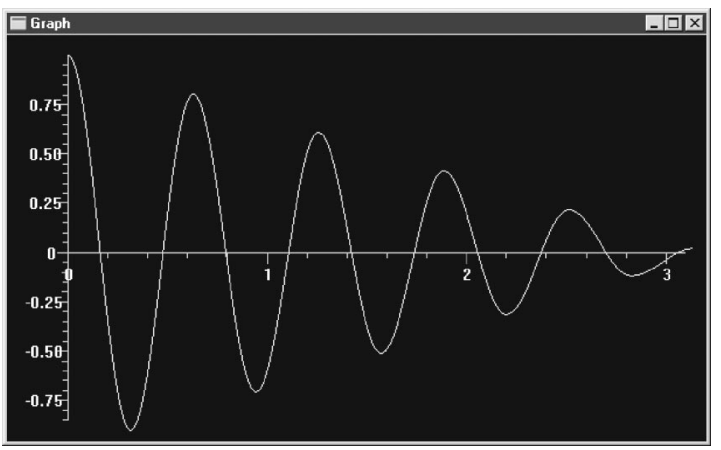

Fig. 6. Position vs time graph produced by the VPython program shown in Fig. 5. The graph and the animation, Fig. 7, are produced simultaneously. 


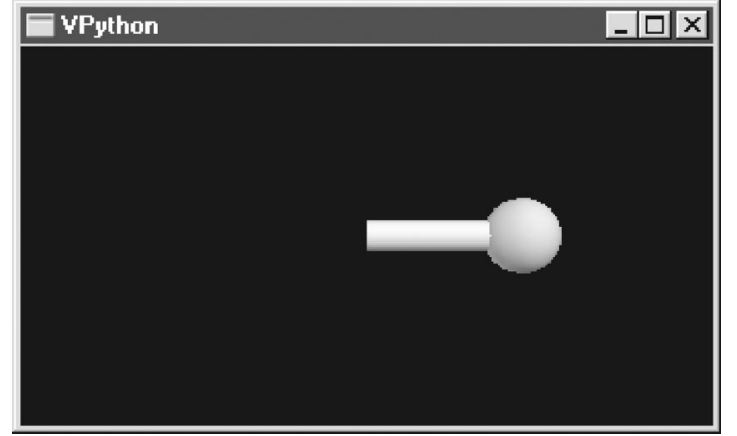

Fig. 7. Screen shot of the animation produced by the program shown in Fig. 5. The block is represented by the sphere object called "block" and the spring is represented by a cylinder object called "spring." The animation window is created when the object block is defined. The block's position and the spring's length are animated by updating the appropriate attributes of each object (block.pos and spring.axis).

because numerical integration of Newton's second law takes center stage in the programs. The VPython program shown in Fig. 5 illustrates the point. The program produces the position versus time graph in one window (Fig. 6) as it generates an animation of the system in another window (Fig. 7). When the motion ceases, the program prints the total distance traveled in a text window. Notice that only a few lines of code are needed to produce the graph and the animation. The basic algorithm shown in the example can be used to solve a wide variety of problems, including many that do not have closed form solutions.

When students can write the same algorithm to study many diverse systems, they quickly begin to appreciate the general applicability of the principle on which the algorithm is based. It is powerful to learn that the same physics can be applied to solve many seemingly different problems, from finding the trajectory of Mars around the Sun to investigating how the speed of a falling raindrop evolves with time.

\section{CONCLUSION}

We have developed two solutions to a seemingly trivial physical situation. The results are physically interesting. When a spring/mass system is damped by a constant magnitude force, the result is an oscillator that decays linearly with time. This behavior is in stark contrast with results for the spring/mass system damped by a force proportional to velocity, which decays exponentially with time. Instead of becoming imperceptibly small, the motion of the oscillator damped by a constant magnitude force ceases completely after a finite time.

In the classroom, the simple spring/mass system damped by a force of constant magnitude turns out to be a rich playground for physics students. It is amenable to analysis by relatively simple algebra as well as a more advanced, calculus-based approach. Simulations can be used in the classroom to help students gain additional insight into the phenomenon. By selecting the approach most appropriate for their students, teachers can present a situation for study that requires deep thinking and cannot simply be looked up in the text.

\section{ACKNOWLEDGMENTS}

The authors would like to thank the US. Department of Education's FIPSE program (P116B71905 and P116B000659) and the National Science Foundation (DUE9752313 and DUE9981107) for their on-going support of the SCALE-UP project. The authors also thank the members of the NCSU Physics Education Research and Development Group, especially Matthew Kohlmyer, who helped us learn VPython.

\section{${ }^{a)}$ Electronic mail: marchew@post.tau.ac.il}

${ }^{b)}$ Electronic mail: David.S.Abbott@Dartmouth.edu

${ }^{c}$ Electronic mail: beichner@ncsu.edu

${ }^{1}$ Richard R. Hake, "Interactive-engagement versus traditional methods: A six-thousand-student survey of mechanics test data for introductory physics courses," Am. J. Phys. 66, 64-74 (1998).

${ }^{2}$ Jeffrey M. Saul, David S. Abbott, Rhett J. Allain, Duane L. Deardorff, and Robert J. Beichner, "Evaluating introductory physics classes in light of the ABET criteria: An example from the SCALE-UP project," Proceedings of the 2000 Annual Meeting of the American Society for Engineering Education, Seattle, WA.

${ }^{3}$ Randall D. Knight, An Instructor's Guide to Introductory Physics (Addison-Wesley, San Fransisco, CA, 2002).

${ }^{4}$ Lillian C. McDermott, Peter S. Shaffer, and The Physics Education Group, Tutorials in Introductory Physics and Homework Package (Prentice-Hall, Upper Saddle River, NJ, 2001).

${ }^{5}$ Patricia Heller and Mark Hollabaugh, "Teaching problem solving through cooperative grouping. 2. Designing problems and structuring groups," Am. J. Phys. 60, 637-644 (1992).

${ }^{6}$ More information on the SCALE-UP project is available at 〈http:// www.ncsu.edu/per $\rangle$.

${ }^{7}$ Interactive Physics, MCS Software, Mandeville, LA. Information about the software can be found at $\langle$ http://interactivephysics.com $\rangle$.

${ }^{8}$ VPython is free and open source. An introduction to VPython can be found at 〈http://vpython.org $\rangle$.

${ }^{9}$ The only book we found that analyzes this system is Douglas A. Quadling and Allan R. D. Ramsay, Introduction to Advanced Mechanics (Bell, London, 1962)

${ }^{10}$ It is tempting to solve for the total distance traveled by equating the initial elastic energy with the product of frictional force and distance. The approach fails, however, because there is no guarantee that the block comes to rest at the spring's equilibrium position.

${ }^{11}$ Bruce Sherwood, "Pseudowork and real work," Am. J. Phys. 51, 597-602 (1983).

${ }^{12}$ A. John Mallinckrodt and Harvey S. Leff, "All about work," Am. J. Phys. 60, 356-365 (1992)

${ }^{13}$ Raymond A. Serway and Robert J. Beichner, Physics for Scientists and Engineers (Saunders, New York, 2000), 5th ed., Vol. 1, p. 133.

${ }^{14}$ The int ${ }^{*}$ function defined here is almost identical to the commonly used int (integer part) function. If $x$ is not an integer, int $*(x)=\operatorname{int}(x)$. If $\mathrm{x}$ is an integer, int $*(x)=\operatorname{int}(x)-1$.

${ }^{15}$ Ruth Chabay and Bruce Sherwood, Matter and Interactions (Wiley, New York, 2002), Vols. 1 and 2. 